\title{
ViMeTGame: A serious game for virtual medical training of breast biopsy
}

\author{
Rafael Siqueira Torres, Helton Hideraldo Bíscaro, Luciano Vieira de Araújo, Fátima L. S. Nunes \\ Laboratório de Aplicações de Informática em Saúde (LApIS) \\ Escola de Artes, Ciências e Humanidades - Universidade de São Paulo (USP) \\ rafael.siqueira.torres@usp.br, heltonhb@usp.br,lvaraujo@usp.br,fatima.nunes@usp.br
}

\begin{abstract}
Serious games can provide an attractive way for training procedures. Some applications for simulating training in the health area are available, but most of them do not include ludic aspects. This article presents a serious games to simulate breast biopsy exam using Virtual Reality. Immersive aspects were included to increase users engagement. An evaluation with users indicated that ludic aspects increase motivation for playing, haptic device was suitable for interaction, colors and illumination techniques were appropriate to provide visual realism and this type of application can present a successful use among students.
\end{abstract}

Keywords-serious games; immersion; virtual reality; medical procedures training; breast biopsy simulation

\section{INTRODUCTION}

Medical training is a subject of several Virtual Reality (VR) researches which aims to improve the user's skills. Serious games have been used to include entertaining aspects in education and training in several situations as organizational procedures, critical situations in companies and business management [1]. Serious games cited in literature related to healthcare use VR techniques and devices to assist in training and education of students as well as rehabilitation of patients. They are useful tools to increase the range of experience with medical situations of students, residents and physicians. The real-time interaction and threedimensionality of the VR allied with entertaining aspects of serious games are a useful combination for building realistic medical training applications to improve clinical skills [2].

Ma and Bechkoum [3] presented a set of serious games to aid the physiotherapy processes in order to improve rehabilitation of patients with motor problems. In one of them, the patient (player) used his hand's movements, captured by a camera, to control a box in Virtual Environment (VE) and reap fruits falling from trees. Jing et al.[4] developed a game for training medical students for identifying bleeding while performing surgery. The authors created a realistic VE and included playful features, as score, time limit and objectives to be achieved.

Petrasova et al. [5] presented a VE that was a serious game for training mothers in care of babies with eating disorders. A wireless controller was used for navigation in VE. The work by Zhang et al. [6] describes the implementation of a virtual marathon using immersive aspects and nonconventional devices. VE aspects included realism, avatars for player's representation and soundtrack referring to the real environment. A marathon treadmill and an exercise bike were used to simulate some tests. Also a camera was included to update the avatar's facial features corresponding to the user's facial features during training.

Others works in literature use VR training applications directly related to training health professionals in medical procedures. Using real Nuclear Magnetic Resonance images, Tang et al. [7] presented a system of virtual neurosurgery, in which the user can navigate in a virtual ventricle using a conventional mouse. Cote et al. [8] provided a simulator of scoliosis surgery in a collaborative and immersive environment with haptic feedback. A system for acupuncture training was presented by Kanehira et al. [9] using a specific device with sensors for simulating the real needle. Despite the realism of these simulators, we do not find entertaining aspects for this type of training in the literature.

In a previous paper, we developed a framework [10] which enables the generation of VR tools for medical training in biopsy, with basic features and functionalities required in this type of simulation. However, such applications do not include playful elements, which can make training more motivating. This article presents an application for medical training based on concepts of serious games and virtual reality whose purpose is to train the ability of insertion of medical instruments for performing breast biopsy examinations.

Besides this introduction, this paper was divided into following sections: section II, which presents basic concepts discussed in the article; section III, which presents the methodology used to develop the application; section ?? presents and discusses the results obtained, and, finally, section $\mathrm{V}$, which presents conclusions about the work.

\section{BACKGROUND}

\section{A. Biopsy exams}

Puncture exams are requested by physicians when there are suspect cases related to anomalies in a patient. They are also known as biopsy exams and can be performed in several human organs. In general, these exams extract little parts of the suspect tissue to send them to laboratory in order to confirm a diagnosis.

Silva [11] mentions that the most used methods to the initial diagnosis of breast cancer are core needle biopsy and 
fine needle aspiration. The former allows the execution of a few invasive procedure as well as allows an analysis about the effect of a nodule on a set of cells (histopathology). The latter allows only the analysis of cells and alterations that they cause in pathological cases (cytopathology).

Additionally, according to Frankel et al. [12], the fine needle aspiration technique presents a more accessible method for physicians and patients, once their complications are rare, the handling of the procedure is simple, and it can be conducted in clinics. Due its character simplified and accessible, this technique was chosen to be simulate in the application presented in this paper.

\section{B. Frameworks}

According to Bosch and Mattson [13], frameworks are sets of classes which promote code reuse. They also provide resources to developers in order to allow the reuse without a deep knowledge of the code.

Johnson and Foote [14] state that oriented-object frameworks enable the reuse of high granularity code. These researchers also stress that the most important characteristic of this frameworks category is the control inversion, which usually is used by developers that desire run a program to call further necessary libraries.

Considering applications for biopsy training based in Virtual Reality, there are several common functionalities to be implemented, as collision detection, deformation, manipulation of medical devices, among others. Presence of commons tasks favors the code reuse and frameworks become an important concept to build applications in this context, as presented in the next section.

\section{Methodology}

The goal of this application is provide an alternative and attractive way to allow the training of the skills necessary to execute a biopsy procedure by students. The target public are undergraduate students, specifically from radiology speciality. The tool also can be used as support in classrooms for teaching the biopsy procedure. However, the main contribution is allow that students feel sensations similar to the real procedures, mainly related to the tact and sequence of tasks necessary for this kind of procedure.

The development of the serious game was divided into the following steps:

1) generation of an initial application using the $\mathrm{ViMeT}$ framework;

2) definition of the game storytelling with aspects found in literature;

3) creation of a database for providing support to the game;

4) inclusion of immersive aspects.

In this section we present the development steps, which from now on will be named ViMeTGame.

\section{A. The ViMeT framework}

The framework used was developed using Java language [10]. It aids in the generation of Virtual Reality applications for medical training, considering specifically the biopsy exams domain, by providing a VE with virtual objects that represent one human organ and one medical device.

VE for simulation can be automatically generated by using a graphical interface where the user (programmer) interacts to choose objects and define parameters desired to the application that will be generated. It is possible define aspects related to visualization and interaction. The source code in Java language, with commands to include the functionalities defined, is provided to programmer who can customize the final application.

It is noteworthy that this framework provides important functionalities for virtual training, such as collision detection with accuracy, deformation of flexible objects in the region of contact with the rigid object, besides interaction with conventional equipments (mouse and keyboard) and nonconventional devices (dataglove and haptic device) [15]. Figure 1 shows some examples of applications generated by $\operatorname{ViMeT}$ framework.

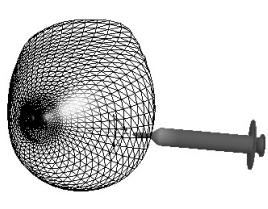

(a)

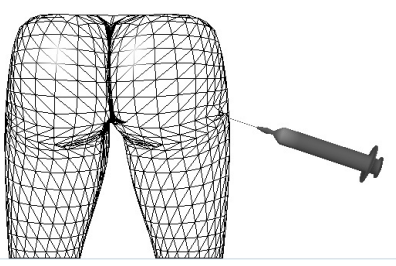

(b)
Figure 1. Examples of applications generated by the ViMeT framework: (a) breast and (b) glutes [10].

An application for simulating breast biopsy was chosen as starting point to ViMeTGame, using initially mouse and keyboard, hierarchical collision detection with Octrees using the method cited in Kera et al.'s work [16], stereoscopic visualization with anaglyphs and mass-spring deformation method [17].

\section{B. Storytelling of the ViMeTGame}

The intention of this application is transform a medical training system for breast biopsy simulation in a more attractive application by using serious games concepts. Thus, the game storytelling was the own biopsy procedure, composed by tasks that user should make during the real procedure, with additional tasks that constitute challenges for the player. In a general way, the player must manipulate a medical instrument (representing a syringe) to puncture the virtual breast. Then, the nodule within the virtual organ must be reached. This procedure is repeated considering five levels 
of difficulty. To access the next level of the game, the player must answer a question randomically retrieved from a database, which concerns the theoretical concepts about the procedure. These tasks are detailed in this section.

When the game starts, the player receives a biopsy request and an image with the nodule position. Based on this information, the player uses a virtual syringe to achieve the hidden nodule. Therefore, the game storytelling is compose of situations related to biopsy procedure. The use of variations on biopsy request or even on the nodule images aims to test the player's ability to perform the procedures planning, e.g. definition of number of samples to be taken or which type of syringe should be used. After this step, the player must perform a biopsy as accurately as possible. At this point, the manual skill of the player is evaluated based on the number of movements made with the syringe, number of samples collected, number of incisions to reach the nodule and speed to perform the procedure. As the last two items are directly related to the safety and comfort of the patient, they are used as the main skills to be achieved for the next game level. Thus, at the end of each game the player will not only tested their ability to define strategies for performing the biopsy but also their ability to accomplish them manually. Moreover, the game's approach allows to organize the tasks to be performed and highlights details that must be done.

The ViMeTGame aspects were defined after a literature revision about studies that use VR and immersive aspects applied to serious games. This process were conduct as a Systematic Review (SR) which synthesizes works published in a particular domain so that it is independent of prejudgment and has scientific value [18]. The SR conducted in this work was described in Torres and Nunes [19].

Playful aspects outlined in the studies included in the SR were considered in the development of the application to promote greater motivation in training by providing challenges to end-users (medical students, residents and physicians). The hypothesis therefore is that the user experience becomes more exciting. Thus, we defined the following aspects:

1) inclusion of virtual object representing a nodule in the inner of the virtual breast, which should be reached by the virtual medical device;

2) inclusion of score to indicate that user is proceeding to hit the target (nodule);

3) definition of time limit for completion of goal;

4) inclusion of soundtrack during the training;

5) inclusion of more realistic visual and interaction aspects in order to enhance the immersion in the game;

6) inclusion of phase with theoretical questions as challenge to transpose to the next level of the game.

With these aspects, the ViMeTGame storytelling was defined as to make the user reaches a nodule (symbolized by a sphere) by using the object that represents the syringe, simulating the biopsy procedure. When performing the task, the student obtains points and can change the game level answering a theoretical question. The points obtained in each phase are accumulative and can be exchanged by tips to help answer the theoretical questions.

The game has 5 difficulty levels, each one with two phases. The first stage of each level provides interaction with the VE to simulate the procedure, as shown in Figure 2. As the player approaches the virtual medical device to the virtual human organ, he scores points. A time limit was set for each level in order to the player fulfills his goal.

The difficulty to performing the procedure increases at each level to make the game challenging. The components used to vary the difficulty consists in changing the position and rotation of the virtual object that represents the breast as well as the size and positioning of virtual object that represents the nodule.

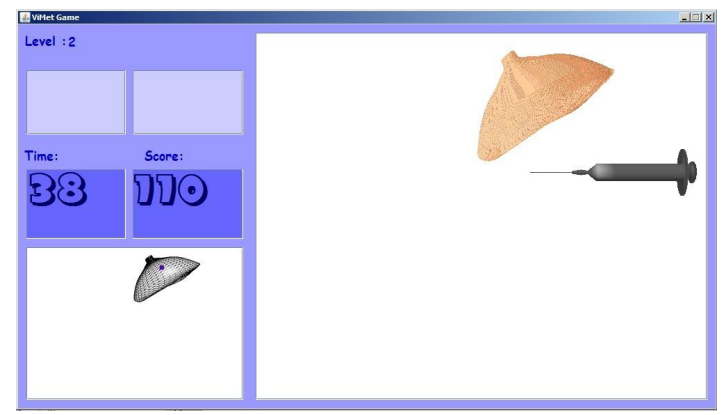

Figure 2. Example of the first phase of a ViMeTGame level

As mentioned, the second stage of each level consists of an objective theoretical question whose correct answer enables the player to transpose the current level and reach the next (Figure 3). Figure 4 shows the state diagram representing the operation of the game.

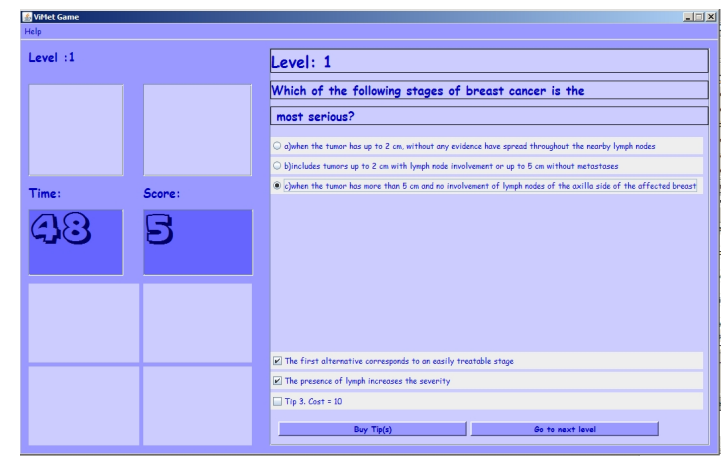

Figure 3. Example of the second phase of a ViMeTGame level.

In the diagram of Figure 4 states "q1", "q3", "q5"and " $\mathrm{q} 9$ " check whether the time has run out. The states "q2" and "q4"check collisions with the nodule and the breast, respectively. The state "q10"verifies if the theoretical question was answered correctly and "q11"advances to the next level. The 


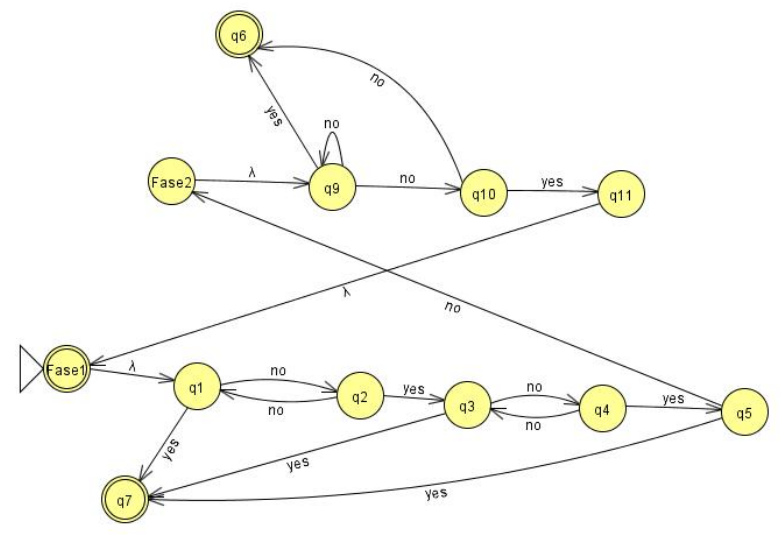

Figure 4. Automaton created to ViMeTGame storytelling.

states "q7"and "q12"spell the end of the game. The state passages "yes" and "no"are the occurrences of the events represented by the states diagram.

All levels have a particular soundtrack, added to increase the user immersion sense. The time limit was included to demonstrate that the procedure can not be delayed because it can cause discomfort in patient. In addition to being an aspect that increases the challenge of training, the score can be used as a measure for assessing the performance of the learner.

The inclusion of menus to help the player has also been implemented, containing information about instructions, the game storytelling and the commands used for navigation and interaction in VE.

\section{Database}

A relational database was implemented in the ViMeTGame aiming at: (1) making it possible the choice of random questions in second stage of each level (phase of theoretical questions previously mentioned) and (2) to store history of the players in order to create a "ranking". Figure 5 shows the logical model of the database.

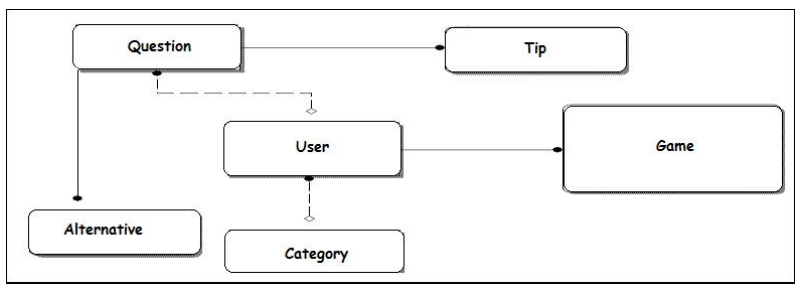

Figure 5. Logical model of the database.

The randomly selection of questions also contributes to make the game more attractive. The inclusion of questions in the database allows classify them in difficulty levels. Thus, the raffle considers questions easier in the early levels and more difficult questions in the final levels. An interface was built in order to allow include questions in the modeled database. This interface is used only by authorized people in the game (teachers). The identification of the user type (player or teacher) is made at the player registration, also using a friendly interface.

To compose a players' training log, data as game's date, final score and spent time, among others, are stored. Thus, both the player and the teacher can compare the players' performance. Figure 6 shows the interface available to display the history of users data.

Additionally, we implemented an interface to display the "ranking" and the other data related to the training of the ten best players every time the user finishes the game (Figure 7).

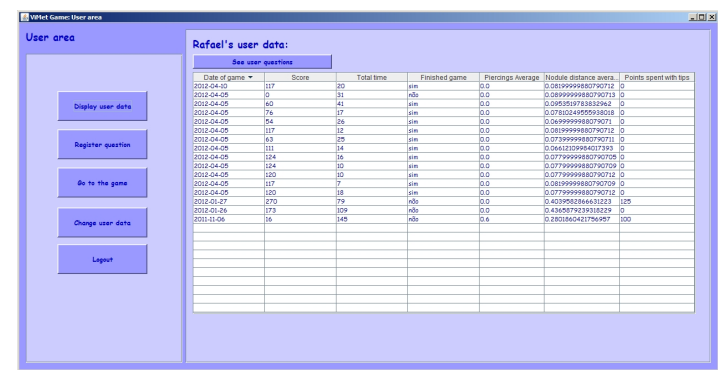

Figure 6. Interface of users' history.

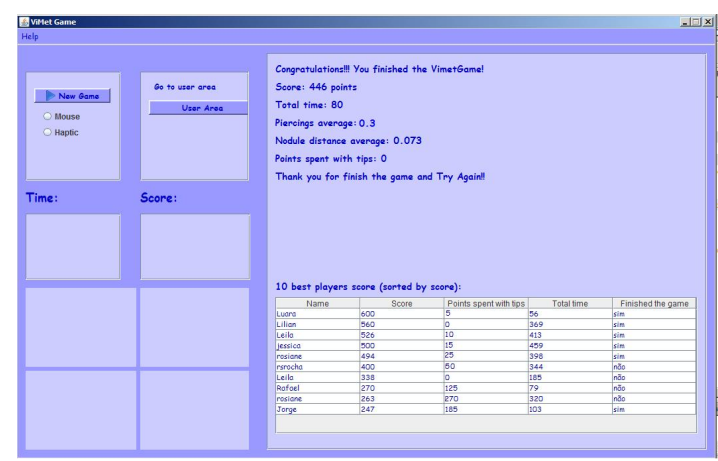

Figure 7. Interface showing the game "ranking".

\section{Collision detection mechanisms}

In order to become more suitable the simulation of biopsy training, the mechanisms of continuous collision detection have been implemented. A collision detection involves checking the time at which occurs a sufficiently small approximation between the objects of an VE in such way to allow overlap between them.

In the ViMeTGame, collisions occur at two moments: when the object that represents the syringe come into contact with the virtual human organ and when the tip of the virtual medical device collides with the object that simulates the nodule. 
At the first collision the Octree technique is used. This technique was provided by the ViMeT framework [16] and consists of a refinement of two other techniques (BoundingBox and BoundingSphere), provided by Java3D API [20]. These two techniques involve the objects in a sphere or in a cube, and check if there is area overlap between them. By the time this occurs a collision between the objects involved is detected.

The Octree technique divides the collision area into octants and then selects the not empty octant that has the smallest Euclidean distance from the center of the colliding object. This process is performed for successive refinements. The BoundingSphere technique is used to wrap each new octant obtained, until it reaches a minimum size (at which time the collision is detected) or it is empty (no collision detected).

The second collision between the syringe tip and the nodule consists in using the same BoundingSphere technique between the virtual nodule and a sphere inserted into the tip of the object representing the medical instrument. This sphere is invisible to the player and was included to provide precision for the collision detection mechanism.

\section{E. Realism and immersive aspects}

In order to increase the sense of immersion and realism perceived by the player, some visual aspects as well as additional interaction aspects have been implemented in ViMeTGame.

It is important to stress that both the time for procedure execution and the precision in the tasks are important in the simulation and consequently in the game. The time is important because it is directly related to the procedure dexterity. In this game, precision is treated by the punctuation and collision detection.

The collision detection provided by the framework used already allows a adequate precision and it was adapted for this game as described in previous section. In order to consider this requirement as a ludic aspect, user obtains points as he/she moves the virtual medical device in direction to the virtual organ and after to the nodule.

The haptic device was chosen as interaction device because user must feel the force feedback when the virtual device touches the objects previously mentioned. Thus, when a collision is detected the player receives a tactile sensation that lets you perceive this action.

Besides these considerations about requirements that must be reached, additional aspects to increase the realism were included, as described following.

The first highlight was designed to amplify the sense of visual realism for players, consisting in the application of realistic color and illumination on the virtual objects. From a survey conducted earlier to determine the influence of these aspects in the perception of realism [21], the game considers the choice of different color and illumination combinations: skin colors "Light", "Brunette"and "Black", besides models of illumination: "Ambient", "Reflective", "Directional"and "Spot"can be combined in order to compose the game. Figure 8 shows some examples of results, which can be compared to the version without the application of these features (Figure 1).

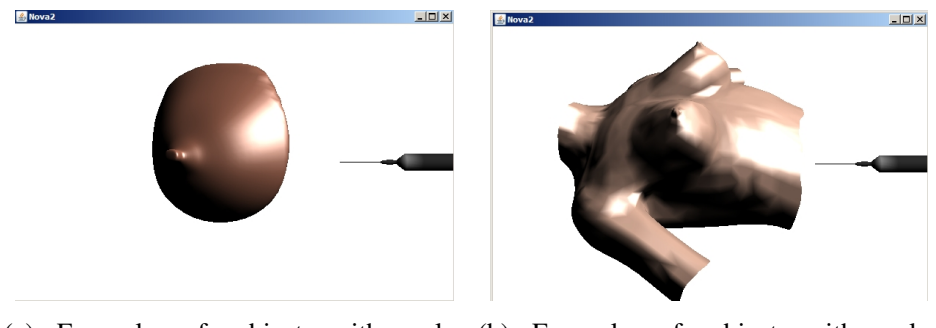

(a) Example of object with color (b) Example of object with color "Brunette"and directional light "Light"e spot light

Figure 8. Example of applications of colocar and illumination models used in ViMeTGame.

Another approach used to increase the realism was the inclusion of levels with objects that provide a sense of depth perception in three-dimensional environment (3D) using the anaglyph technique. This technique can provide a simple way to cause the depth sense through the superimposition of two images of different colors, but with a displacement between them. Figure 9 shows the result of applying the technique in the game.

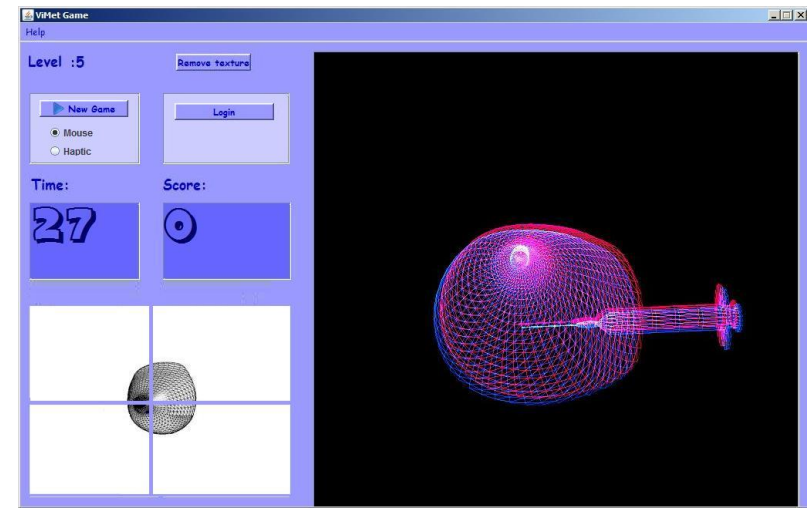

Figure 9. Level of the game with visualization using anaglyph.

In the actual breast biopsy procedure, the physician can be guided to the correct location observing the two views from X-ray mammographic images (craniocaudal and mediolateral). Thereby, to increase the realism another implementation included, in some game levels, mammographic images in order to reproduce the biopsy procedure more accurately. At these levels, the virtual three-dimensional object available for training corresponds to two-dimensional images available. Objects were simulated by a system which analyzes mammography images and creates a virtual object corresponding to a particular case, considering both the 
breast contours and the nodule shape present in the twodimensional images [22].

Figure 10 shows the level of the game that offers artistically modeled objects. In Figure 11 the three-dimensional object faithfully represents the images of mammograms provided at the bottom left.

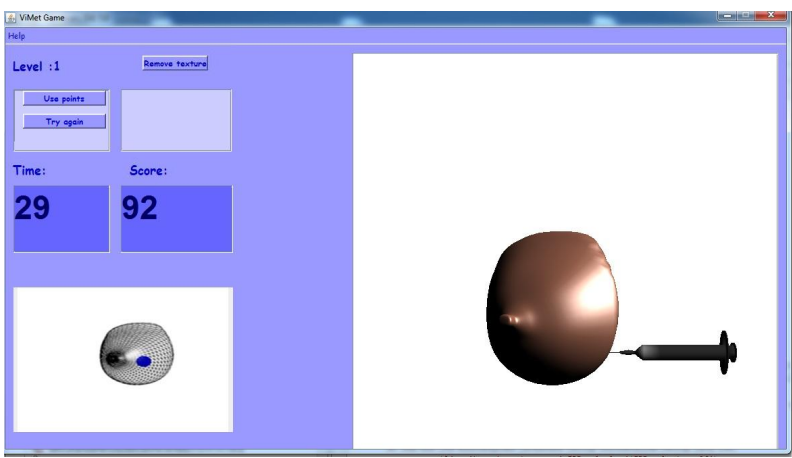

Figure 10. Level of the game with artistically modeled objects.

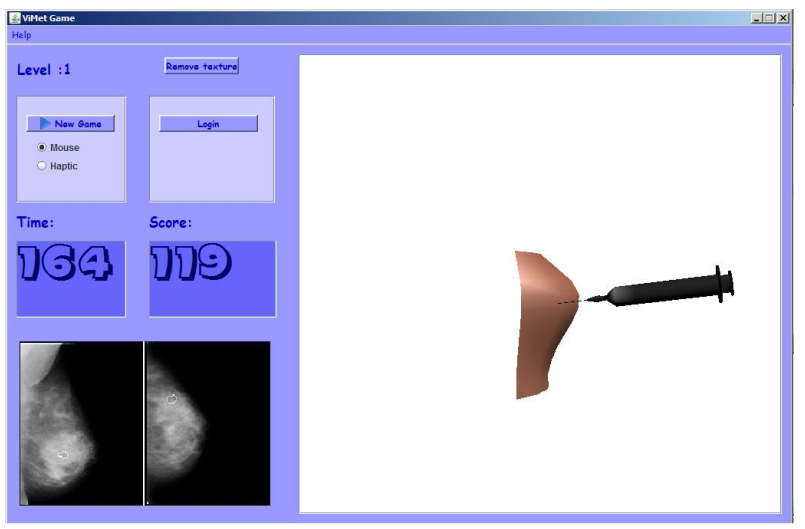

Figure 11. Level of the game with realist objects.

Regarding the interaction, the main aspect implemented in ViMeTGame to increase immersion was the inclusion of the haptic device (Figure 12). Such device was included as a mouse substitute, previously used for navigation in the VE. The device is used to control the virtual object that simbolizes the syringe. When a collision occurs between the virtual medical instrument and the virtual nodule, the player receives a force feedback. The haptic device returns information to the application such as position on each axis, force, state of the buttons and frequency of communication with the device.

\section{Results And Discussions}

The goal of the evaluation presented in this Section was to verify the immersive aspects of the application by comparing the resources used to interaction and visualization. The adequacy in relation to the functionalities and goals of the

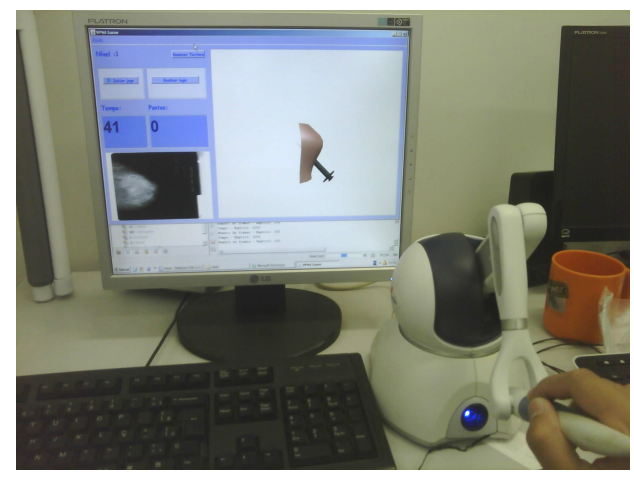

Figure 12. Execution of the game with the haptic device.

tool (improve skills students and act as support in classroom) was not within the scope of this assessment.

The ViMeTGame evaluation was conducted in two steps: first, a pre-evaluation performed with computer students to investigate the application's gameplay and second, a final evaluation performed with students and health professionals. The pre-evaluation, described in [19], work as a fine adjustment step in the game difficulty, helping to set up the times limit and score. Moreover, in this step it was possible to identify the need of including immersive aspects in the game since the visual features of the objects and the mouse interaction have not proved satisfactory.

The evaluation did not consider the phase with theoretical issues due to tailor the experiment to the data acquisition. This procedure avoided that the user looses the game before pass through all the levels.

Five levels containing all the used approaches were defined. One level uses realistic objects, all the others use modeled objects. Two levels have provided objects using anaglyph and stereoscopy techniques. All the other levels have used only colors and illumination as visual aspects.

The volunteers were submitted to an experiment with four tasks: (1) answer a pre-test questionnaire; (2) play a game of the ViMeTGame using the mouse as input device to handle the virtual objects; (3) play a game using the haptic device as data input and finally (4) answer a post-test questionnaire.

\section{A. Volunteers characteristics}

The serious game's evaluation was performed with ten volunteers (students), of which eight was female and two male aged among 20 and 30 years. Six volunteers were from health care area, and the others were students from other areas. All them signed a consent form stating they are according to the research terms. The evaluators were chosen considering volunteering and availability criteria.

The pre-test questionnaire contained questions that evaluate the knowledge and experience of the volunteers in virtual environments, medical procedures and devices usage. The users also were requested to indicate the presence of vision problems. If so, they should state which was the problem. 
Of the ten volunteers, $50 \%$ claim to have vision problems. Figure 13 shows the indicated problems, being that some of the volunteers have indicated more than one problem.

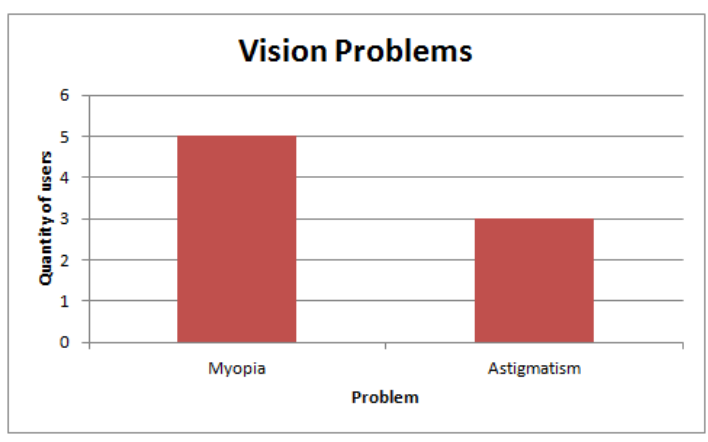

Figure 13. Vision problems declared by the respondents.

The graphics of the Figures 14 and 15 present the volunteers situation over the virtual learning environments experience and the three-dimensional virtual environments. As can be seen, most of the volunteers were inexperienced with respect to such environments.

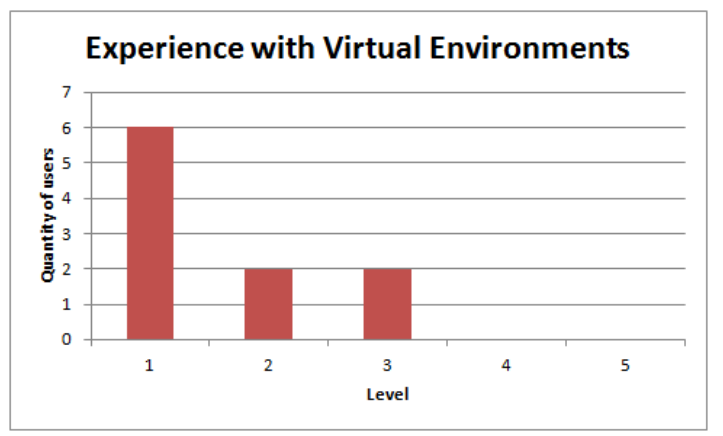

Figure 14. ViMeTGame Evaluation question about virtual learning enviroments experience. Scale: 1 to "Laic"and 5 to "Experienced".

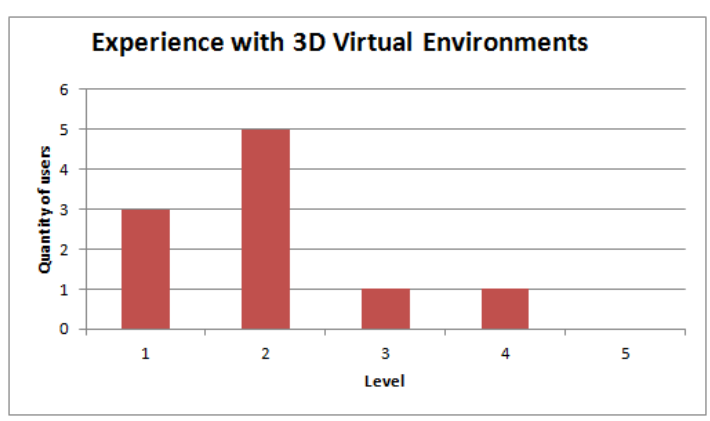

Figure 15. ViMeTGame Evaluation question about virtual 3D experience. Scale: 1 to "Laic"and 5 to "Experienced".

About $80 \%$ of the respondents claim not to have a knowledge about biopsy, and $20 \%$ who have some knowledge (the lowest level marked was 2) belong to health area. With respect to the experience in puncture procedures, half of the volunteers claim not to have a knowledge. From the others $50 \%, 30 \%$ consider themselves experts (Figure 16).

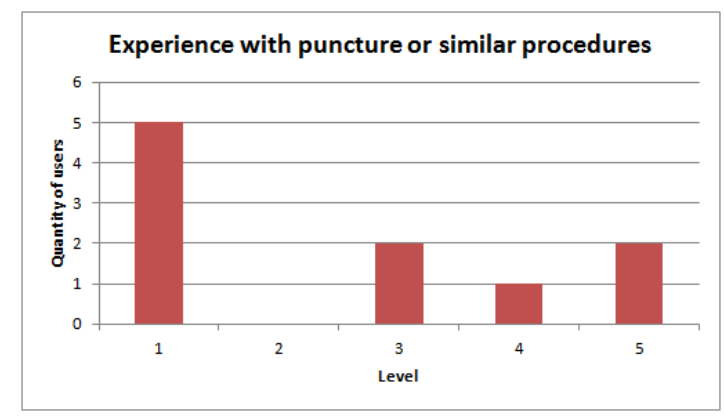

Figure 16. ViMeTGame Evaluation question about the level of knowledge in puncture procedures or similar procedures. Scale: 1 to "None" and 5 to "Expert".

The last question in the health context was about the level of knowledge in breast diseases. The result was $20 \%$ for each level range. If we consider that from level 3 is related the good knowledge, $60 \%$ of the volunteers reached this mark as can be seen in Figure 17.

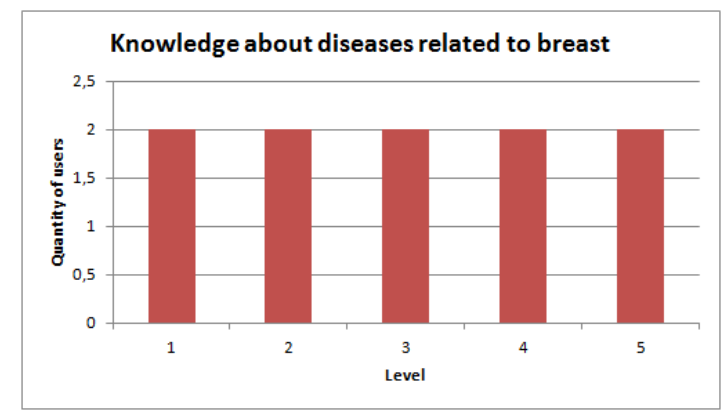

Figure 17. ViMeTGame evaluation question about the level of knowledge brest diseases. Scale: 1 to "None"and 5 to "Expert".

Finally, the last question in the pre-test questionnaire asked the opinion about the use of computational tools to help in the training of medical professionals to perform surgical procedures as biopsy. The group answer with highest score on the scale. About $90 \%$ of the respondents indicate that this type of computational tool can be useful to medical training. Figure 18 illustrates this answer.

\section{B. Visual aspects and interaction}

After this pre-test quiz, the volunteers played two matches in the serious games, the first using the haptic device and the second using the mouse to control the virtual syringe. In order to familiarize themselves with the objects movements, the volunteers were instructed to play a demo version, which consist in handle the virtual syringe using the both devices in an application generated by the ViMeT framework, without the game goals and aspects. The students also were instructed to sign up for their data to be stored and the score was displayed in the "ranking". 


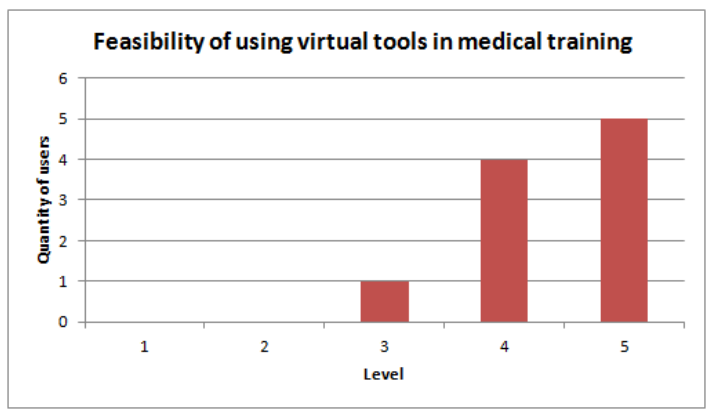

Figure 18. ViMeTGame evaluation question about using computational tools in medical training. Scale: 1 to "Do not believe"and 5 to "Totally believe".

The main goal of the post-test quiz is to evaluate the gameplay and the immersive aspects offered by the game. When they were about to play a level in which would be need glasses for 3D viewing, the respondents were instructed to use it by the observer.

In relation to the depth sensation provided by the anaglyph technique, in general, the volunteers did not realize the depth sensation. Those who declare not to feel any depth sensation amounted $20 \%$ as well as those who attribute the second lowest note of the scale. Another $40 \%$ have attribute the average grade of the scale, which means that they perceived the depth in a limited way. The last $20 \%$ claim full depth sensation.

The results referred to the depth sensation could be related with the number of the volunteers that declare to have vision diseases. From five volunteers that claim to have vision diseases, four have attribute values among 1 to 3 in the scale to this question. When comparing to the other five which has no vision problems, all of them have attribute scores among 3 to 5 , which may suggests that the anaglyph technique was damaged. Another interesting fact is that all the health area volunteers have attribute the highest score in this question. Figure 19 presents this results.

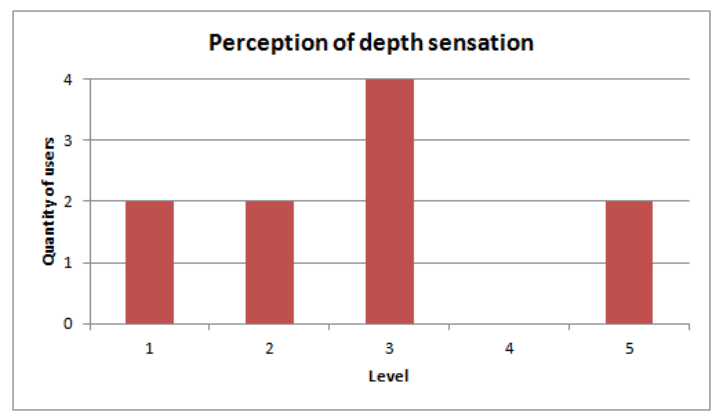

Figure 19. ViMeTGame evaluation question about the depth sensation experienced in the game. Scale: 1 to "None"and 5 to "Full sensation".

When not used anaglyph technique, volunteers were asked if the objects have colors resembling human skin. Although nobody has assigned the highest score, $50 \%$ of the volunteers gave a 4 and $30 \%$ attributed the average grade. This result hints that the objects displayed with colors and illumination techniques provided a good realism perception to the volunteers (Figure 20).

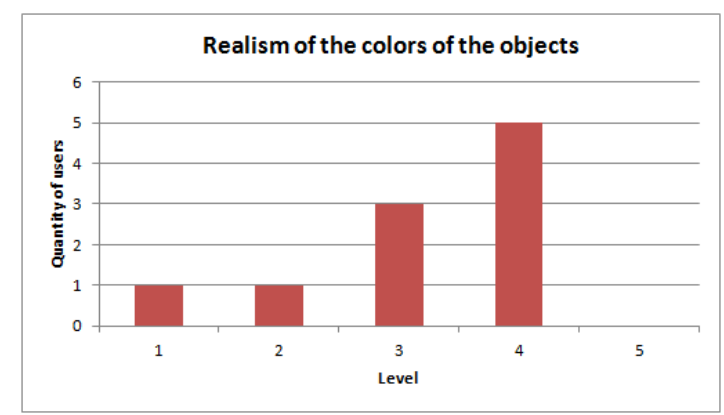

Figure 20. ViMeTGame evaluation question about color realism of the virtal objects. Scale: 1 to "Little similar"and 5 to "Very similar".

About using the haptic device, $60 \%$ of the volunteers have assigned the two highest scale score, while $20 \%$ have preferred the average score. The remaining $20 \%$ have attributed the two lowest score. It can be observed that most of the volunteers could feel the realism provided by the device. It is worth mentioning that in the $20 \%$ that gives the lowest score, $50 \%$ was those that have attribute lowest score with relation to mouse experience. This could characterize a difficulty navigating in three-dimensional environments due to lack of experience. The same $20 \%$ have attribute the lowest score in the question about three-dimensional virtual environments experience.

The volunteers also were questioned if the mouse could adequately substitute the haptic device. Even with $40 \%$ of the volunteers not recognizing the haptic device as a tool that allows the object manipulation in a realistic way, $80 \%$ of the volunteers said that the mouse can not nicely replace the haptic device. Therefore, this result suggests that the haptic device inclusion improves the realism sensation in the object's motion (Figures 21 and 22).

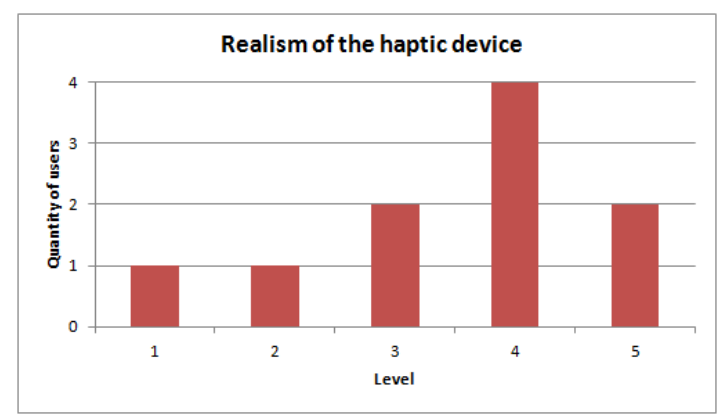

Figure 21. ViMeTGame evaluation question about the realism level of the haptic device. Scale: 1 to "None"and 5 to "Full sensation". 


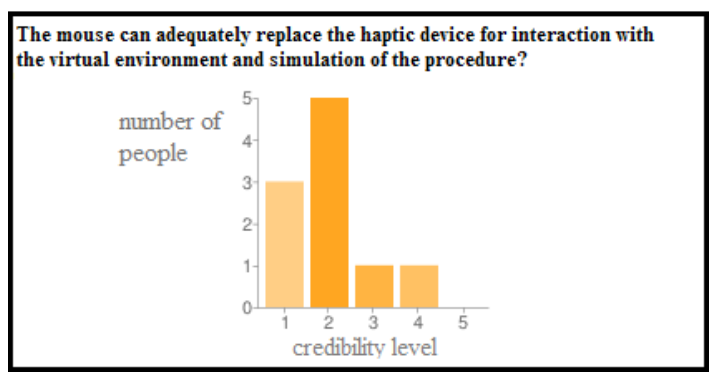

Figure 22. ViMeTGame evaluation question about the mouse usage. Scale: 1 to "Disagree"and 5 to "Fully agree".

\section{Gameplay aspects and training}

The first gameplay aspect evaluated was the time to conclude the tasks. About $60 \%$ of the volunteers have assigned the two highest score in the scale, $20 \%$ have gave the score 3 and the remaining have attributed 2. Therefore, in this considered group, no user considered the time as insufficient. It is important to note the importance of a previous evaluation before the application reach the final user. The adjustments made after the first evaluation [19] helped make these aspects more appropriate.

Another observation concerning the time tasks is that $60 \%$ of the volunteers claim that have felt pressured with the timing provided in the interface, and $90 \%$ of them have attribute the highest value in the scale. Furthermore, 20\% of the volunteers have attribute the average score and the remaining have attribute the two lowest scores. This suggests that the time task provides a challenge to the player, and could contribute to the player motivation. This element is appropriate to the present work because it refers to durations of the medical procedure. Figures 23 and 24 illustrate the data for those questions.

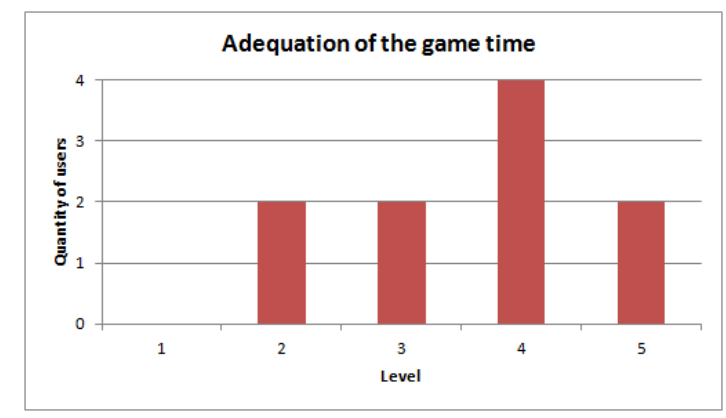

Figure 23. ViMeTGame evaluation question about the timing of the game. Scale: 1 to "Disagree"and 5 to "Fully agree".

About the game, the volunteers were asked if the level of difficulty was increasing in each level. About $60 \%$ of the volunteers have attribute the score of 4 to this question. From the remaining 40\%, 20\% have attribute the average score and the remaining have indicate the two lowest score in the scale. This hints that the group of evaluators have

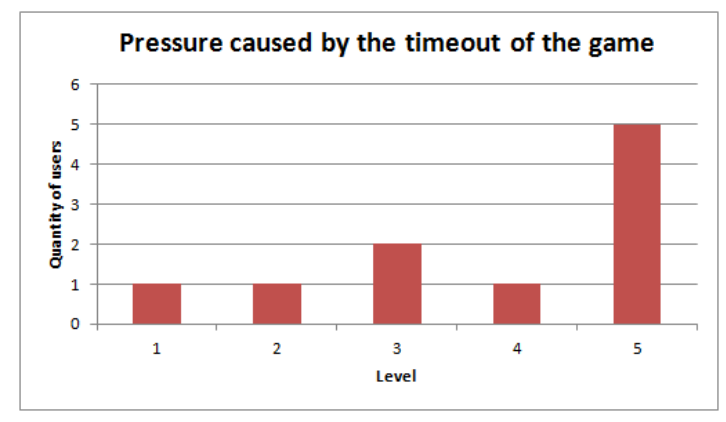

Figure 24. ViMeTGame evaluation question about pressure that the time could cause. Scale: 1 to "Disagree"and 5 to "Fully agree".

notice the increasing level of difficulty, which collaborates with the player motivation (Figure 25).

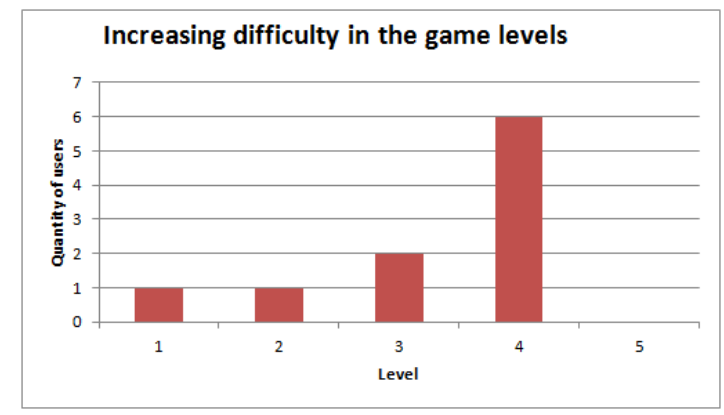

Figure 25. ViMeTGame evaluation question about the game dificulty. Scale: 1 to "Disagree"and 5 to "Fully agree".

It was also investigated if the ranking availability is a motivating factor. About $50 \%$ of the volunteers have attributed the two highest scores of the scale, and the remaining $30 \%$ have attribute the average score (Figure 26). Thus, this may mean that the ranking motivates players to competition.

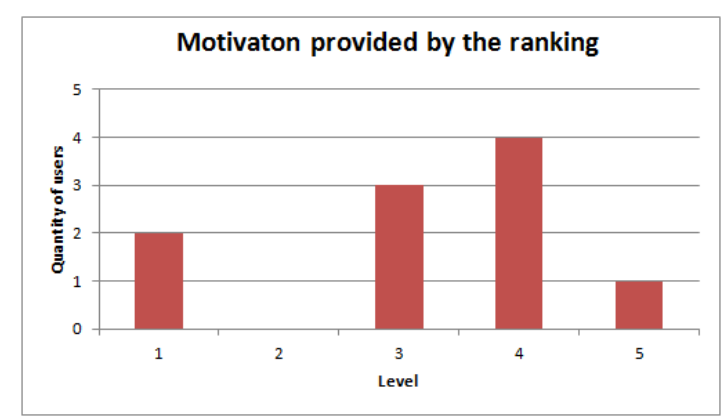

Figure 26. ViMeTGame evaluation question about the ranking. Scale: 1 to "Disagree" and 5 to "Fully agree".

About the soundtrack, $60 \%$ of the volunteers said that the music have contributed to keep the game attention. From the $40 \%$ remaining, $70 \%$ have given the second lowest score, which can suggest that the soundtrack does not refers to the real environment, and may not be ideal for this kind 
of application. It is worth to add that no volunteers have attributed the lowest score in the scale, which hints that the soundtrack was not reproved by the players. The graphic in the Figure 27 illustrates the results for this question.

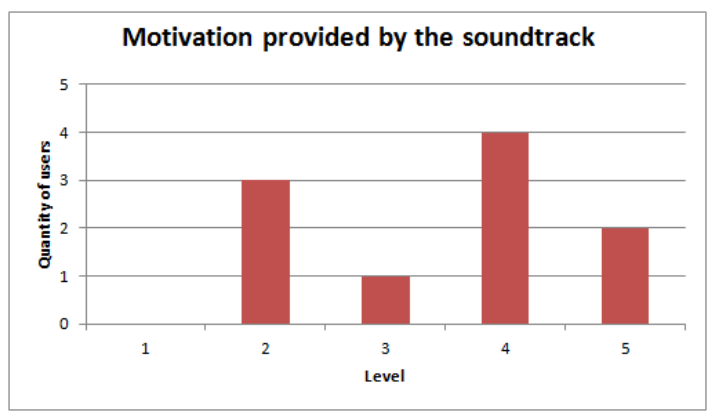

Figure 27. ViMeTGame evaluation question about the soundtrack. Scale: 1 to "Disagree"and 5 to "Fully agree".

The latter two questions were about the game experience. The first one asked the volunteers if the use of a game for this type of training showed motivating. The answers show a high level of satisfaction among the volunteers.All users have attribute scores 4 and 5, suggesting that the serious game can constitute an important tool not only to teaching, but also to training.

The last question tried to relate the visualization methods and the devices, asking what was be the best approach to provide a realistic sensation as well as the sensation of "be within the environment". The options were "mouse + color and illumination", "mouse + anaglyph", "haptic + color and illumination" and "haptic + anaglyph". The volunteers was divided about this question. About 50\% of answers pointed to the option "haptic + color and illumination"and the remaining marked "haptic + anaglyph"as can be seen in Figure 28.

As no approach was unanimous, it is not possible to state that one approach emerges above the other. It was expected that the anaglyph version would provide a bigger realism sensation, but this result can be due to the difficulties aforementioned. It is possible to compare the answers with respect to the field volunteers. Those pertaining to health area, $70 \%$ preferred the approach "haptic + anaglyph", and the players that have attribute the highest score to the anaglyph approach were all of the health field. It can be hint that for users of the health area, this is a more interesting approach.

No volunteers preferred a mouse approach, which suggests that the haptic device is really a better approach for this kind of application. It is worth to mention that the approach that uses color and illumination techniques can be a good option since it has a minimum of realistic appearance of objects.

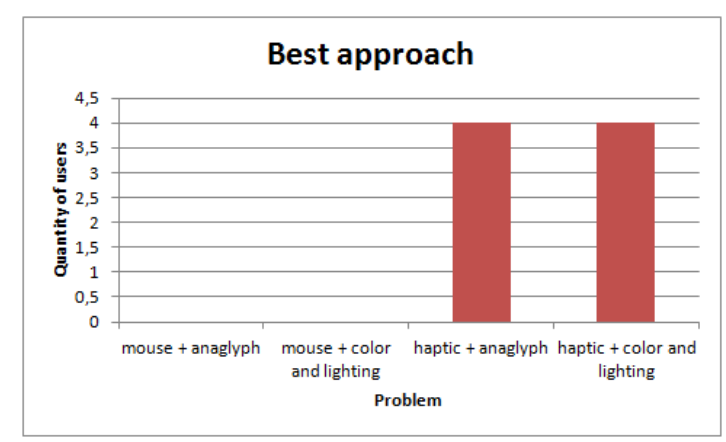

Figure 28. ViMeTGame evaluation question about the best approach used in the experiment.

\section{CONCLUSions}

This paper presented the development and evaluation of a VR application as a serious game to enable the training of breast biopsy exam in order to improve clinical skills.

The main contribution of this paper was the development of a VR Breast Biopsy game. In order to improve the user experience and learning when using the 3D training software, this game was designed to explore several features, such as: use of haptic device to enhanced the sensation of manipulate a real syringe, development of collision techniques to provide force feedback when the needle touches the nodule, definition of realistic objects based on real mammographic images, applying realistic color and illumination, creation of game storytelling based on breast biopsy cases, definition and implementing of a database to historical data storage and register of questions, implementing of ranking and raffle of questions.

By the evaluation with users, we observed that the realism in virtual objects helps in the user's immersion. However, the group which evaluated the game noticed a difference in preference in relation of the screen: while health professional players have preferred the stereoscopic anaglyph preview version, players from other areas have indicated as a better version without anaglyph, using only the colors and illumination in three-dimensional objects.

We also found that the availability of a ranking of the players, time limits to perform the task and scores to the user who hits the goal of the training with the application worked as challenges, included entertaining aspects of this type of acquisition skill.

We think other types of evaluation must be conducted, mainly with professor to verify the effectiveness of the tool in relation to improve students skills and aid in the teaching process. Even though the evaluation could be continued by including additional evaluators, the amount of users is consistent with the type of evaluation conducted, according to works related in literature. The evaluation conducted showed that some aspects were fully satisfactory and others no nice to the players. The use of haptic device was very highly 
rated and regarded as optimal for this type of applications, fulfilling the objective of increasing the immersion.

\section{REFERENCES}

[1] L. S. Machado, R. M. Moraes, F. L. S. Nunes, and R. M. E. M. Costa, "Serious games baseados em realidade virtual para educação médica," Revista Brasileira de Educação Médica, vol. 35, pp. 254-262, 2011.

[2] N. P. Karunasekera, "Effectiveness of virtual reality based immersive training for education of health professionals: a systematic review," Master Thesis, University of Canterbury, New Zeland, 2011

[3] M. Ma and K. Bechkoum, "Serious games for movement therapy after stroke," in Systems, Man and Cybernetics, 2008. SMC 2008. IEEE International Conference on, oct. 2008, pp. $1872-1877$.

[4] J. Qin, Y.-P. Chui, W.-M. Pang, K.-S. Choi, and P.-A. Heng, "Learning blood management in orthopedic surgery through gameplay," Computer Graphics and Applications, IEEE, vol. 30, no. 2, pp. 45 -57, march-april 2010.

[5] A. Petrasova, S. Czanner, A. Chalmers, J. Farrer, and D. Wolke, "The playability evaluation of virtual baby feeding application," in Second International Conference on Games and Virtual Worlds for Serious Applications (VS-GAMES), 2010, pp. 95-100.

[6] M. Zhang, M. Xu, Y. Liu, G. He, L. Han, P. Lv, and Y. Li, "The framework and implementation of virtual network marathon," in International Symposium on VR Innovation (ISVRI). IEEE, 2011, pp. 161-167.

[7] C.-Y. Tang, W. Chin, Y.-P. Chui, W.-S. Poon, and P.-A. Heng, "A Virtual Reality-based Surgical Simulation System for Virtual Neuroendoscopy," in Integration Technology, 2007. ICIT '07. IEEE International Conference on, 20-24 2007, pp. 253-258.

[8] M. Cote, J.-A. Boulay, B. Ozell, H. Labelle, and C.-E. Aubin, "Virtual reality simulator for scoliosis surgery training: Transatlantic collaborative tests," in Haptic Audio visual Environments and Games, 2008. HAVE 2008. IEEE International Workshop on, vol. 1, no. 1, 18-19 2008, pp. 1-6.

[9] R. Kanehira, A. Shoda, M. Yagihashi, H. Narita, and H. Fujimoto, "Development of an Acupuncture Training System Using Virtual Reality Technology," in Fuzzy Systems and Knowledge Discovery, 2008. FSKD '08. Fifth International Conference on, vol. 4, 18-20 2008, pp. 665-668.

[10] A. C. M. T. G. Oliveira and F. L. S. Nunes, "Building a open source framework for virtual medical training," Journal of Digital Imaging, vol. 23, pp. 706-720, 2010.

[11] A. Ribeiro-Silva, "Core biopsy: uma técnica confiável para o diagnóstico histopatológico do câncer de mama?" Jornal Brasileiro de Patologia e Medicina Laboratorial (Impresso), vol. 48, pp. $8-9,2012$.
[12] P. P. Frankel, V. F. Esteves, L. C. S. Thuler, and R. J. da Silva Vieira, "Acurácia da punção aspirativa por agulha fina e da punção por agulha grossa no diagnóstico de lesões mamárias," Revista Brasileira de Ginecologia e Obstetrícia, vol. 33, pp. 139 - 143, 2011.

[13] J. Bosch and M. Mattsson, "Framework composition: Problems, causes and solutions," in Proceedings of TOOLS International Conference on Technology of Object Oriented Systems and Languages. Santa Barbara, CA, USA: IEEEComputer Society, 1997, pp. $203-214$.

[14] R. E. Johnson and B. Foote, "Designing reusable classes," Journal of Object Oriented Programing, vol. 1, no. 2, pp. 22 $-35,1988$.

[15] F. L. S. Nunes, H. H. Biscaro, M. E. Delamaro, R. Tori, and R. Nakamura, "Lapis: developing research applied to health care area," SBC Journal on 3D Interactive Systems, vol. 2 , pp. 63-66, 2011.

[16] M. Kera, H. Pedrini, and F. L. S. Nunes, "Ambiente virtual interativo com colisão e deformação de objetos para treinamento médico," Revista de Informática Teórica e Aplicada (Impresso), vol. 18, pp. 1-10, 2011.

[17] A. C. M. T. G. Oliveira, L. Pavarini, F. L. S. Nunes, L. C. Botega, D. J. Rossato, and A. Bezerra, "Virtual reality framework for medical training: implementation of a deformation classa using java," in ACM International Conference on Virtual Reality Continuum and Its Applications. Hong Kong: Proceedings of ACM International Conference on Virtual Reality Continuum and Its Applications, 2006. v. 1., 2006.

[18] B. Kitchenham, "Procedures for performing systematic review," Keele University, Technical Report TR/SE-0401, 2004

[19] R. S. Torres and F. L. S. Nunes, "Applying entertaining aspects of serious game in medical training: Systematic review and implementation," in XIII Symposium on Virtual Reality (SVR), 2011, pp. 18-27.

[20] Sun, "Java 3D API Tutorial," disponível em:< http://java.sun.com/developer/onlineTraining/java3d/>. Acesso em: ago. 2012.

[21] L. F. Brunialti and F. L. S. Nunes, "Métodos de iluminação e aplicação de texturas para obtenção de realismo em treinamento médico virtual," in 19o. Simpósio Internacional de Iniciação Científica da USP, vol. 1, São Carlos - SP, 2011, pp. 1-1.

[22] S. R. Delfino and F. L. S. Nunes, "Geração de estudos de caso para treinamento médico virtual a partir de técnicas de realidade virtual e processamento de imagens," in Anais do IX Workshop de Informática Médica, Bento Gonçalves - RS, Brazil, 2009. 